\title{
Phase Separation in Ti-6Al-4V Alloys with Boron Additions for Biomedical Applications: Scanning Kelvin Probe Force Microscopy Investigation of Microgalvanic Couples and Corrosion Initiation
}

\author{
P.H. DAVIS, ${ }^{1}$ K. ROBLES,${ }^{2}$ K. LIVINGSTON,${ }^{1}$ S. JOHNS,${ }^{1}$ V.A. RAVI,${ }^{2,3}$ \\ E. GRAUGNARD, ${ }^{1}$ and M.F. HURLEY ${ }^{1,4}$ \\ 1.-Micron School of Materials Science and Engineering, Boise State University, Boise, ID 83725-2090, \\ USA. 2.-Department of Chemical and Materials Engineering, California State Polytechnic University, \\ Pomona, Pomona, CA 91768, USA. 3.—e-mail: vravi@cpp.edu. 4.—e-mail: mikehurley@boisestate.edu
}

To investigate the effect of boron additions on the corrosion behavior of Ti-6Al-4V for potential use in biomedical implants and devices, cast samples of Ti-6Al-4V were alloyed with $0.01 \%$ to $1.09 \%$ boron by weight and subjected to hot isostatic pressing. Subsequent analysis via scanning Kelvin probe force microscopy and scanning electron microscopy/energy-dispersive spectroscopy revealed the presence of both alpha $(\alpha)$ and beta $(\beta)$ phase titanium, enriched in aluminum and vanadium, respectively. At all concentrations, boron additions affected the grain structure and were dispersed throughout both phases, but above the solubility limit, needle-like TiB structures also formed. The TiB needles and $\beta$ phase exhibited similar surface potentials, whereas that of the $\alpha$ phase was found to be significantly lower. Nevertheless, when subjected to high applied electrochemical potentials in saline solutions, corrosion initiation was observed exclusively within the more noble $\beta$ phase.

\section{INTRODUCTION}

Titanium and its alloys are used in numerous applications, ranging from aerospace and high-end bicycles to dental and biomedical implants. In regards to biomedical applications, these alloys have been used as replacements for hip and knee joints, as well as for components in artificial hearts and pace makers. ${ }^{1}$ Commercially pure titanium (CP Ti; UNS R50400) is used in dental applications such as crowns and bridges, as well as for components such as screws ${ }^{2}$ whereas UNS R56400 (Ti-6Al-4V; Ti64) is used as a structural biomaterial for orthopedic prostheses. ${ }^{1}$ The success of these alloys in the medical field is a result of their excellent corrosion resistance, biocompatibility, ${ }^{2-4}$ high strength, and lower Young's modulus in comparison with other implant alloys such as stainless steels and cobalt-chromium alloys. ${ }^{3}$

At room temperature, titanium alloys can exist in either of two phases, a stable hcp alpha $(\alpha)$ phase or a metastable bcc beta $(\beta)$ phase, or a combination of the two. While the $\alpha$ phase is stronger, the $\beta$ phase is more ductile, and accordingly, many titanium alloys are designed to contain a mixture of both phases to optimize alloy properties for the intended application. The Ti64 alloy is composed of an aluminum-rich $\alpha$ phase and a vanadium-rich $\beta$ phase. Even though this alloy has achieved remarkable success as an implant material, it would be desirable to increase its useful life span in light of the projected increase in human life expectancy. ${ }^{5}$ A potential alternative based on a Ti64 matrix is described in this article.

Boron additions to Ti-6Al-4V have been shown to increase yield and tensile strengths of the alloy with the likely mechanism being grain size reduction of the alloy. Dramatic decreases in grain size have been reported even for small amounts of boron additions, i.e., in the $0.01 \mathrm{wt} . \%$ to $0.1 \mathrm{wt} . \%$ range. ${ }^{6}$ When boron additions exceed the solubility limit, titanium monoboride (TiB) precipitates are formed. 

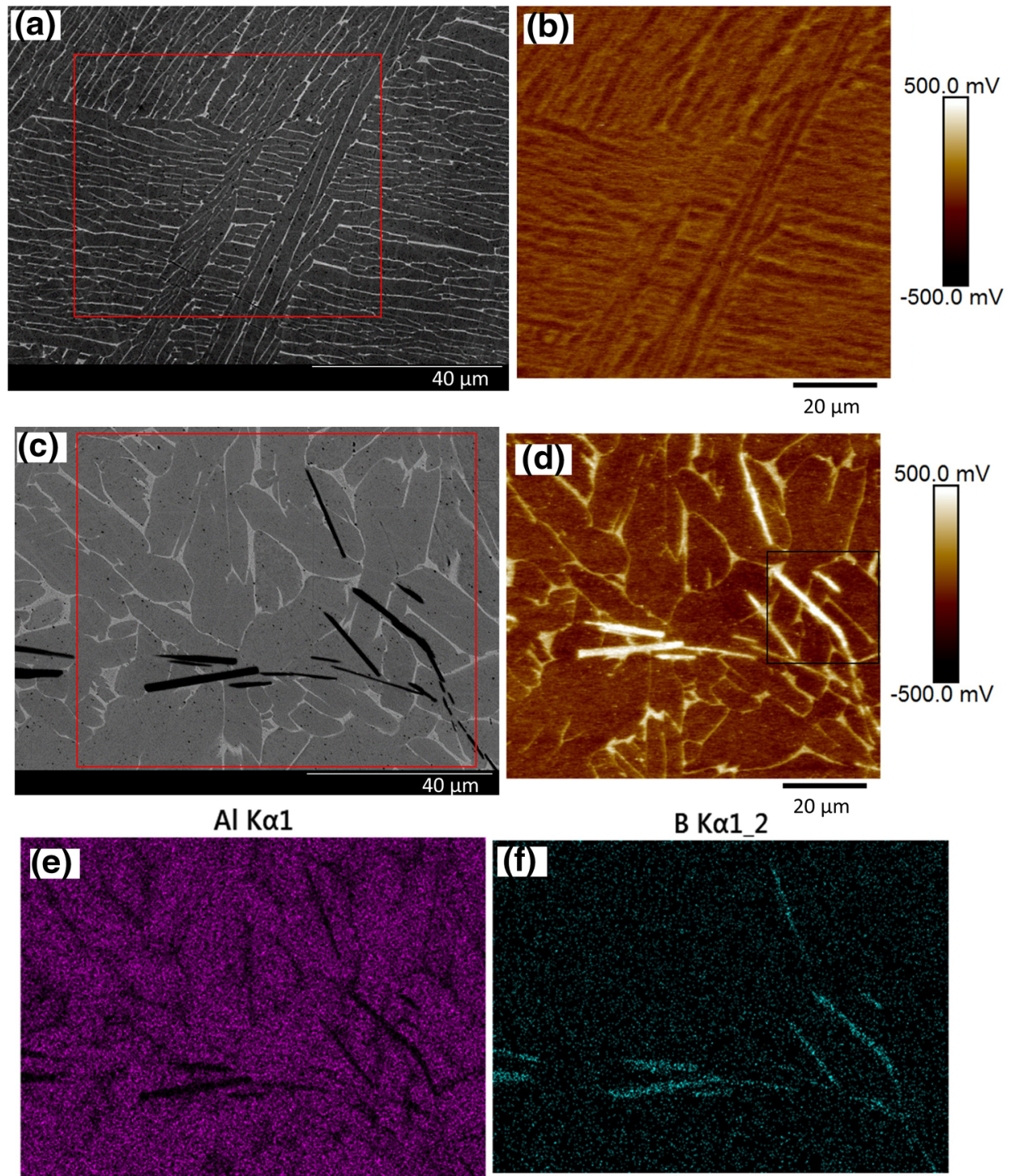

Fig. 1. Co-localized backscattered electron (BSE) SEM (a) and SKPFM (b) images of Ti-6AI-4V alloyed with $0.01 \%$ B showing extensive grain refinement. The smaller grain size leads to reduced contrast between the $\alpha$ and $\beta$ phases in the SKPFM image as a result of spatial averaging. Co-localized BSE SEM (c) and SKPFM (d) images of $0.43 \% \mathrm{~B}$ alloyed Ti-6Al-4V showing the formation of boron-rich needles, per corresponding EDS maps of $\mathrm{Al}(\mathrm{e})$ and $\mathrm{B}(\mathrm{f})$. Red boxes in the SEM images indicate the approximate corresponding location of SKPFM, and the small black box in $d$ shows the representative area for the line scan analysis presented in Fig. 4.

Previous work determined that with the addition of low levels of boron, particularly up to $0.02 \mathrm{wt} . \% \mathrm{~B}$, there is an increase in the corrosion resistance. ${ }^{7-9}$ Nevertheless, the contribution of $\mathrm{TiB}$ to the driving force for microgalvanic corrosion relative to the $\alpha$ and $\beta$ phases has not yet been determined. We have used a combination of nanoscale imaging techniques to elucidate composition, structure, and resultant galvanic potential differences between the microstructural phases present in Ti-6Al-4V alloy samples alloyed with varying weight percentages of boron. Here, we report the results of these studies in an effort to understand the effect of TiB ceramic precipitates on localized corrosion initiation in Ti$6 \mathrm{Al}-4 \mathrm{~V}$.

\section{MATERIALS AND METHODS}

\section{Alloys and Sample Preparation}

Test coupons were machined out of cast and hot isostatically pressed (HIPped) cylinders of Ti-6Al$4 \mathrm{~V}$ alloyed with $0.01 \%$ to $1.09 \%$ boron by weight. ${ }^{7}$ The coupons were metallographically prepared by sequential grinding down to 800 grit, sonication in deionized (DI) water followed by polishing with 6$\mu \mathrm{m}, 3-\mu \mathrm{m}$, and $1-\mu \mathrm{m}$ diamond slurries (Allied) using lapping oil as a lubricant. After again sonicating in DI water, a $0.05-\mu \mathrm{m}$ aqueous alumina slurry was used for final polishing, followed by sonication in DI water and acetone. Each of the sonication steps were carried out for $3 \mathrm{~min}$. After preparation, the 


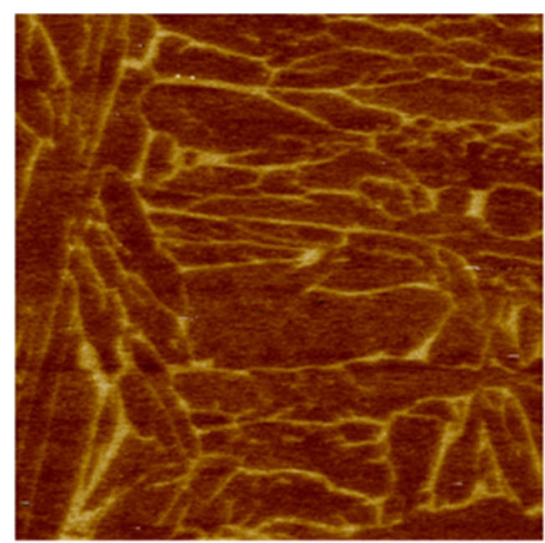

$0.001 \% \mathrm{~B}$

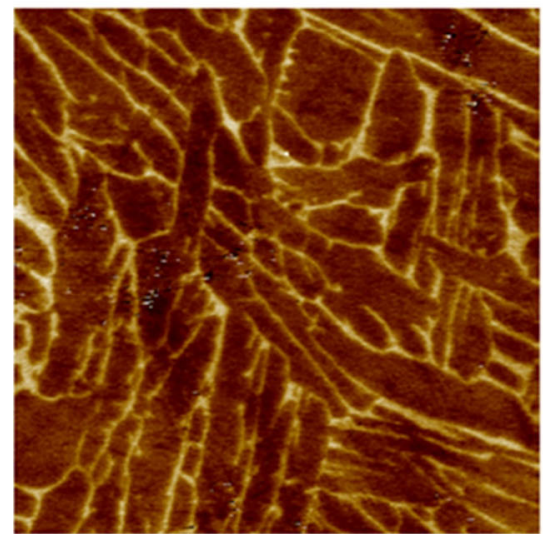

$0.04 \% \mathrm{~B}$

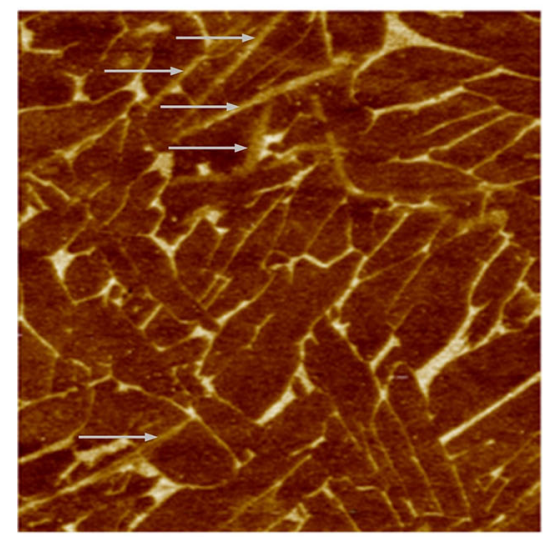

$0.5 \% \mathrm{~B}$
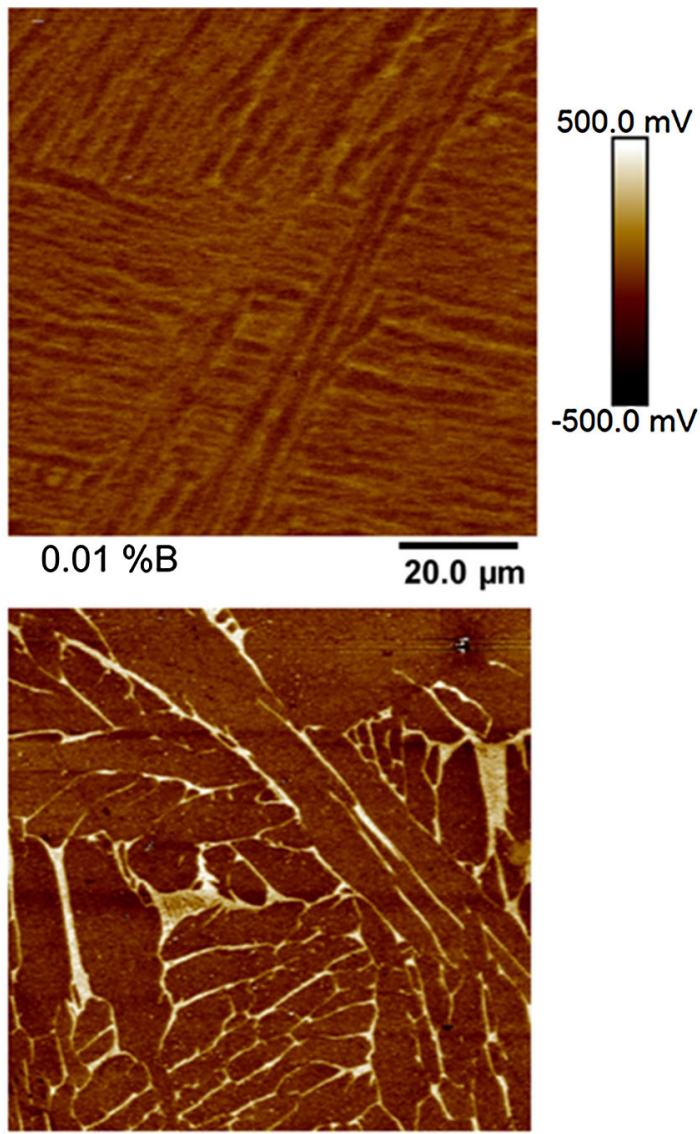

$0.1 \% \mathrm{~B}$

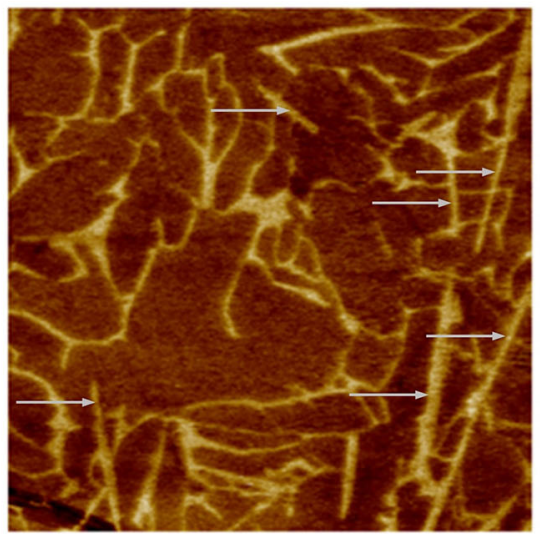

$1.09 \% \mathrm{~B}$

Fig. 2. SKPFM Volta potential maps of boron alloyed Ti-6Al-4V alloys. Boron concentrations shown range from $<0.001 \% \mathrm{~B}$ to $1.09 \% \mathrm{~B}$. Saturation occurs at $>0.02 \% \mathrm{~B}$, and accordingly, samples with higher boron concentrations display formation of TiB "needles" (representative formations indicated by arrows, confirmed via EDS) that exhibit a Volta potential value noble to the surrounding $\alpha$ phase matrix but similar to that of the $\beta$ phase.

coupons were subjected to electrochemical testing in an artificial physiological electrolyte (modified Hanks Balanced Salt Solution) following the ASTM F2129-15 test method for determining the corrosion susceptibility of small implant devices. In addition, alloy coupons were subjected to high peak potentials in saline solutions to examine differences in their corrosion behavior (described elsewhere). ${ }^{7-9}$

\section{Scanning Kelvin Probe Force Microscopy (SKPFM)}

SKPFM imaging was conducted using a Bruker Dimension Icon AFM operating in frequency modulation (FM) PeakForce KPFM mode. ${ }^{10-12}$ After polishing, each test coupon was cleaned with UHP $\mathrm{N}_{2}$ prior to imaging to remove any loose surface 

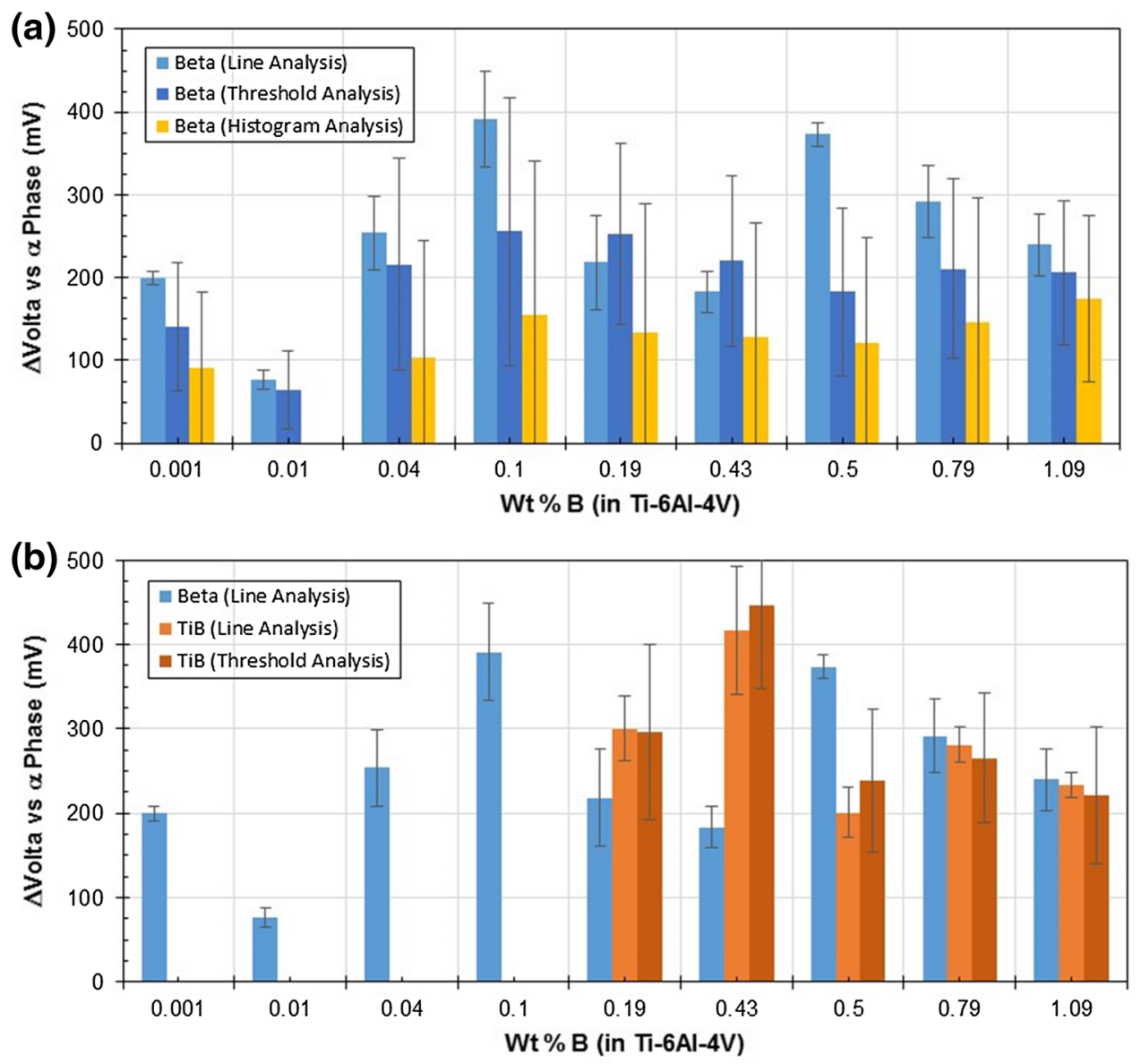

Fig. 3. Volta potentials of the beta phase (a) and boron-rich precipitates (b) relative to the alpha phase as a function of wt.\% B. The apparent reduction in potential difference between alpha and beta phases at low boron concentrations is likely a result of grain refinement and SKPFM spatial resolution limits. Volta potential differences were calculated using several different evaluation methods as explained further in the text and supplementary materials.

particulates. A PeakForce amplitude of $\sim 75 \mathrm{~nm}$ and a lift height of $\sim 85 \mathrm{~nm}$ were used to acquire the topography and surface potential images, respectively. The same PFQNE-AL probe (Bruker) was used to image all samples. Image processing was performed in NanoScope Analysis V1.80, and the resultant surface potential histograms were exported for quantitative analyses.

\section{Scanning Electron Microscopy (SEM)}

SEM imaging was performed in a Hitachi S$4300 \mathrm{~N}$ equipped with a tungsten filament electron source. In addition to standard secondary electron (SE) imaging, backscattered electron (BSE) imaging was also conducted at $10 \mathrm{keV}$. Energy-dispersive xray spectroscopy (EDS) maps were obtained at $15 \mathrm{keV}$. Z-contrast in BSE imaging mode permitted ready identification of $\mathrm{TiB}$ precipitates, whereas EDS was used to construct elemental maps to differentiate between the Al-rich $\alpha$ phase and the V-rich $\beta$ phase. All SEM imaging was carried out after SKPFM because of the potential for carbon deposition by the electron beam, which has been shown to affect the measured surface potentials. ${ }^{10,13}$

\section{RESULTS AND DISCUSSION}

\section{Effect of Boron on Microstructure}

As shown in Fig. 1a and b, at low concentrations, particularly $0.01 \% \mathrm{~B}$, the addition of boron to Ti$6 \mathrm{Al}-4 \mathrm{~V}$ can lead to significant grain size refinement (cf. Figs. 1c, d and 2). The resultant microstructure is composed primarily of $\alpha$ phase grains, interspersed with a fine, interconnected network of $\beta$ phase. The spatial resolution of SKPFM is dependent on probe lift height, and hence, it is related to the inherent surface roughness of the sample. This can lead to spatial averaging of the measured surface potential across submicron-wide microstructural features such as the fine $\beta$ phase network surrounding the $\alpha$ phase grains. This averaging effect is readily seen by comparing the Volta potential contrast for the $0.01 \%$ B sample, which exhibited the smallest grain size (and hence greatest fraction of $\alpha-\beta$ interface) with that seen for other boron concentrations (SKPFM images in Figs. 1b, $d$ and 2). At higher B concentrations, a precipitated $\mathrm{TiB}$ phase appears in the alloy microstructure, characterized by needle-like or blocky structures 

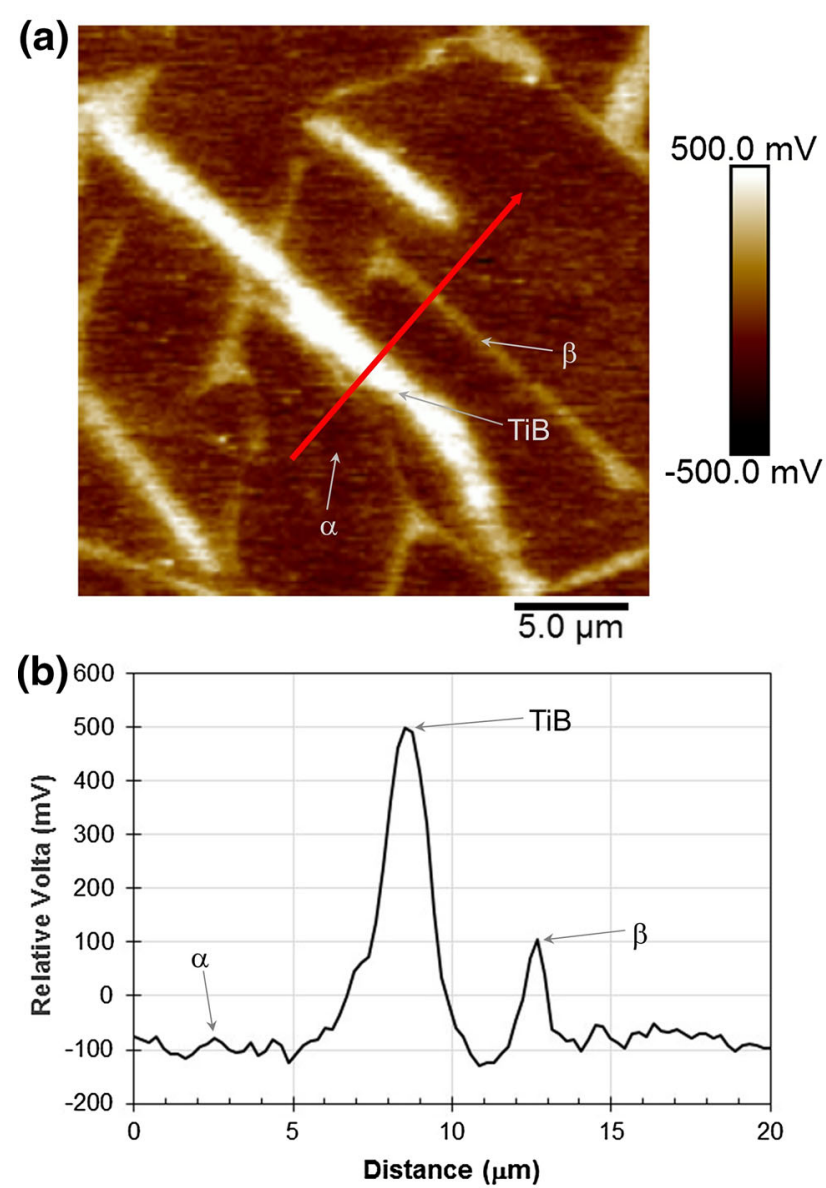

Fig. 4. Volta potential map (a) and corresponding line scan analysis (b) of the $\alpha, \beta$, and TiB phases (black arrows) present in the microstructure of the $0.43 \% \mathrm{~B}$ sample. The red arrow in (a) indicates where the Volta potentials, plotted in (b) as a function of distance were acquired.

tens of micrometers long that are randomly distributed and aligned within the $\alpha+\beta$ superstructure. In Fig. 1c, this TiB phase can be seen as black needles in the BSE SEM image, verified via EDS (Fig. 1f). As shown in Fig. 1d, the resulting surface potential is slightly more positive than that of the $\beta$ phase.

\section{Phase Composition and Volta Potentials}

Figure 2 presents representative SKPFM images of Ti-6Al-4V samples containing varying amounts of added boron. At boron concentrations greater than the saturation limit of $\sim 0.02 \mathrm{wt} . \%$, blocky or needlelike TiB phase precipitates were observed and confirmed through EDS analysis. Although the $\mathrm{TiB}$ precipitates are present in the higher $\mathrm{B}$ concentration samples of Fig. 2, they are not readily distinguishable in most SKPFM images because their relative Volta potential and aspect are similar to that of the $\beta$ phase. Instead, it is necessary to correlate features seen in the SKPFM images with co-localized BSE SEM images and EDS maps, as shown in Fig. 1e and f, to verify phase identity. Colocalized BSE SEM and SKPFM images with corresponding EDS maps for all nine sample compositions studied $(<0.001 \%$ to $1.09 \%$ B) are shown in Figs. S1 to S9 in the supplementary materials.

Likely sites of corrosion initiation can be investigated by considering the microgalvanic corrosion driving force between microstructural features. Compositional and structural differences between adjacent phases and precipitates give rise to local surface (Volta) potential differences. These Volta potentials typically correlate with solution potential and, hence, the expected development of local cathodic and anodic sites between coupled microstructural features. ${ }^{14,15}$ To determine the nature of expected microgalvanic couples within Ti-6Al-4V + B samples, the relative Volta potentials of the $\alpha, \beta$, and $\mathrm{TiB}$ phases were determined from the SKPFM data. The resultant values, calculated via several methods for comparison, are shown graphically in Fig. 3.

Except for the $0.01 \%$ B sample, where extensive grain refinement greatly diminished the potential contrast between the $\alpha$ and $\beta$ phases in the KPFM image, histograms of the potentials recorded from each sample exhibited a bimodal distribution. The histograms were analyzed in two ways to attempt to determine the relative potentials of the phases present (Fig. 3a). First, a threshold voltage located midway between the two peaks of the distribution was selected, with the average potential and standard deviation calculated for the two resultant populations. Second, per the method of Nakhaie et al., the histograms were fit to a sum of two Gaussians to attempt to determine the relative potentials of the phases. ${ }^{16}$ The fits and corresponding results are shown in Fig. S24 and Table S1 of the supplementary materials. Bimodal distributions were observed for even the high wt.\% B samples as the small area fraction of boron-rich precipitates and the similarity of their Volta potentials to that of the $\beta$ phase made them difficult to distinguish based solely on the histogram data. Thus, as another way to determine the Volta potentials of the $\beta$ phase and TiB precipitates relative to the surrounding $\alpha$ phase matrix, line scan analysis was conducted to determine the potential difference between selected microstructural phase features (whose composition was confirmed via co-localized BSE and EDS; see Figs. S1 to S9) and the surrounding $\alpha$ phase matrix as shown in Fig. 4 and Figs. S10 to S23 in the supplementary materials. Selected areas containing only $\alpha$ phase and boron-rich precipitates were also cropped and subjected to threshold analysis as described earlier (see Fig. 3b). Fitting of the corresponding histograms was attempted, but the paucity of boron-rich precipitate data points in any given region made such fitting untenable. The relative merits of these various analysis methods are discussed in greater detail in the supplementary material, but note that all three methods are likely 

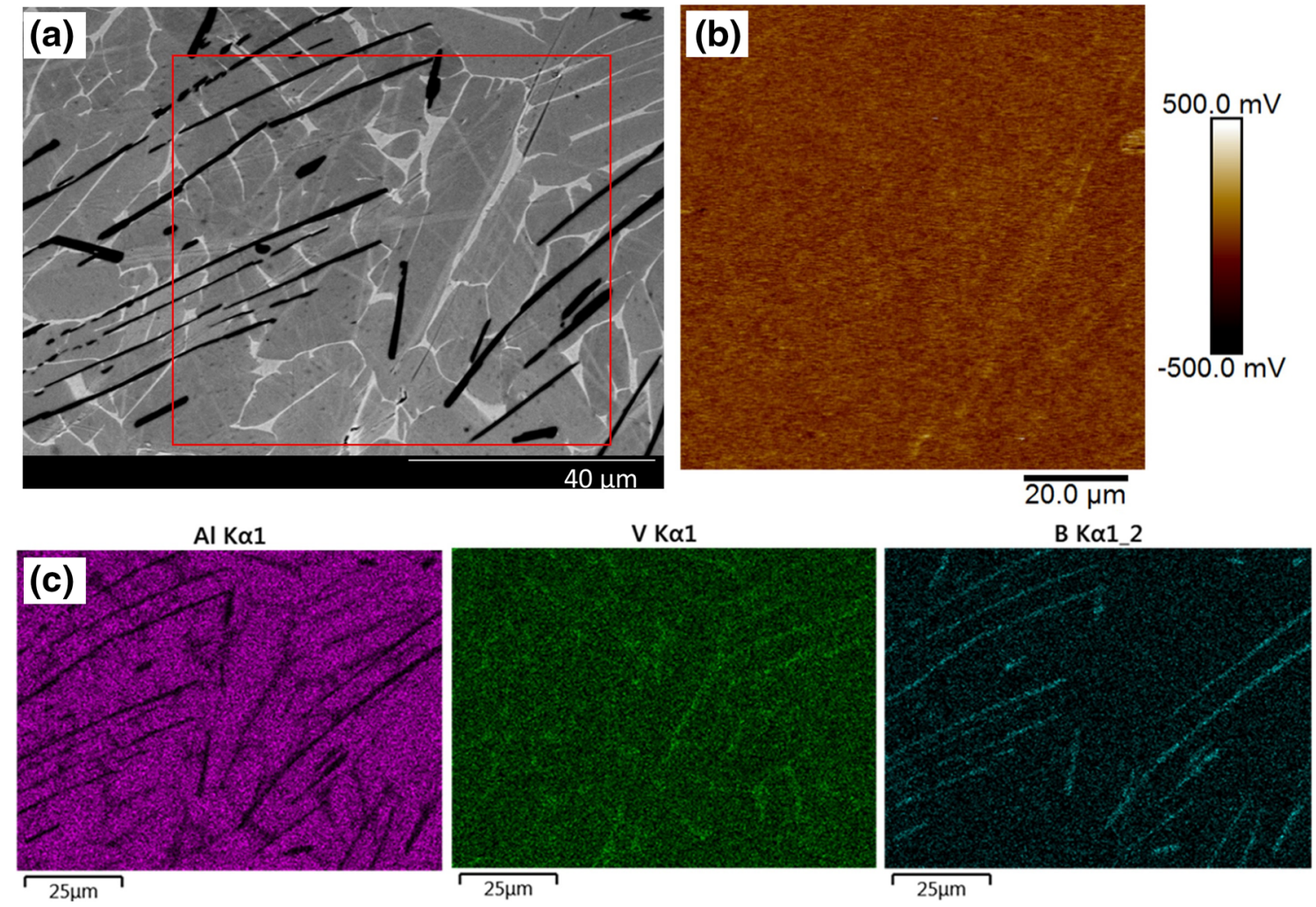

Fig. 5. SKPFM image (b) of a representative Ti-6Al-4V + 1.09\% B sample subjected to the ASTM F2129-15 test protocol. The formation of a thin passivating layer resulted in a more uniform surface potential as measured by SKPFM compared with untested samples (cf. Fig. 2). Co-located SEM (b) and EDS (c) confirmed the phase composition of the microstructure beneath the passive film and lack of evident corrosion attack. Red box in the SEM image (a) indicates the approximate corresponding location of SKPFM image (b).

to underestimate the potential difference between phases as a result of spatial averaging, and thus, the values reported are best interpreted as lower limits on the true potential difference between the microstructural phases present.

The V-rich $\beta$ phase was found to be noble relative to the $\alpha$ phase and possessed a Volta potential $\sim 200-400 \mathrm{mV}$ higher than the $\alpha$ phase depending on the sample. The direction of galvanic coupling is in agreement with that of unalloyed (i.e., boron-free) Ti-6Al-4V. Nevertheless, the results reported here indicate a significantly higher potential difference between the $\beta$ and the $\alpha$ phase than previously reported by others, perhaps because of higher spatial resolution in the current SKPFM scans resulting in less averaging of the measured potentials near phase boundaries. ${ }^{16}$ This averaging effect occurs regardless of the method used to determine the potential difference between phases (e.g., fitting of histograms versus cross-sectioning of selected features; see Figs. S10 to S24 and Table S1) but can be somewhat counteracted in the case of crosssectional analysis, which uses the potential measured at the interior of the phase in preference to that at the phase boundary (and hence is to be preferred in terms of giving a more accurate reflection of the true interphase potential difference). The observed difference in potential between $\beta$ and $\alpha$ phases suggests that when corrosion occurs under natural conditions, the lower relative potential of the $\alpha$ phase will make it the expected anode site, thereby experiencing preferential dissolution supported by cathodic activity within the more noble $\beta$ phase.

Because B has a very low solubility limit in titanium, it is expected that the phase composition of $\alpha$ and $\beta$ are independent of B content and, hence, that the Volta potentials are minimally affected. Thus, although B additions and the amount of cold working can significantly impact the mechanical behavior of Ti-6Al-4V alloys, neither is significantly detrimental to the resulting Volta potential difference between the $\alpha$ and $\beta$ phases or overall corrosion resistance of the alloy. ${ }^{16}$

The relative Volta potential of the TiB phase is higher than that of the $\alpha$ phase, indicating it could act as a preferential cathode during corrosion in addition to (or in preference to) the $\beta$ phase. Specifically, the potential difference implies there is a thermodynamic driving force to favor preferential dissolution of $\alpha$ phase supported by coupled reduction reactions on adjacent TiB phases, unless 
(a)

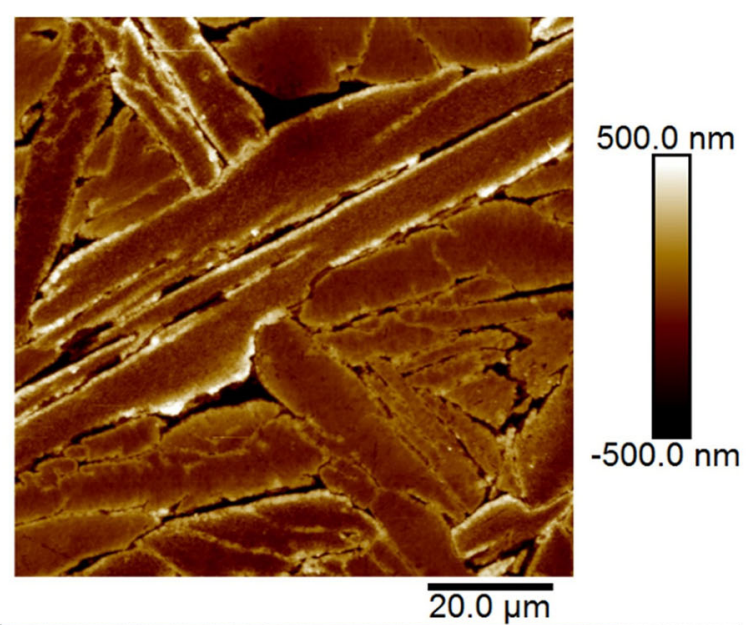

(c)

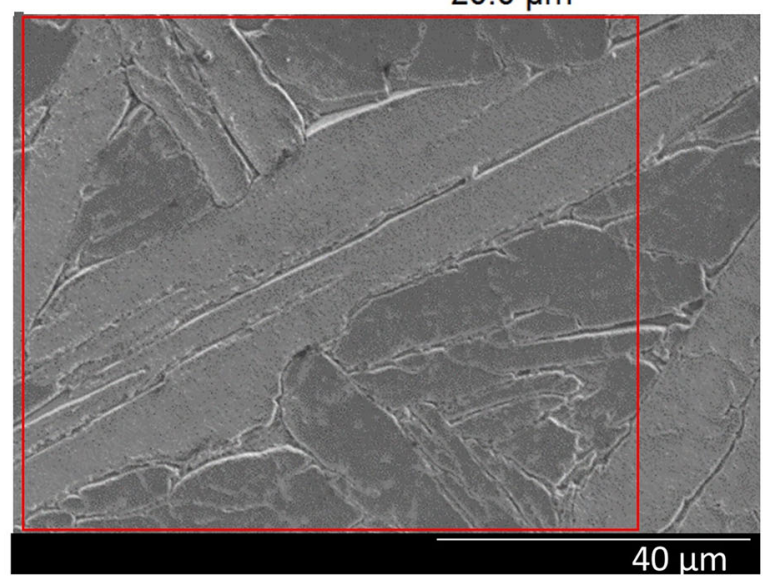

(e)

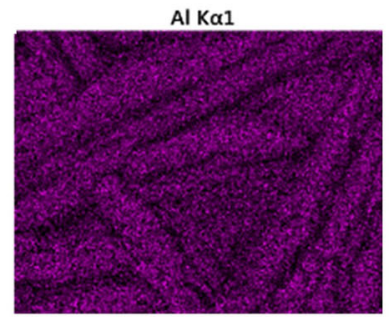

(b)

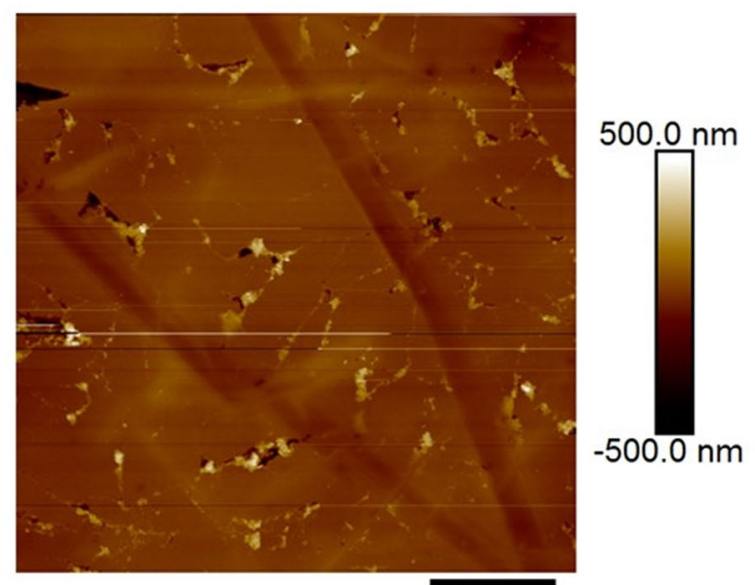

(d)

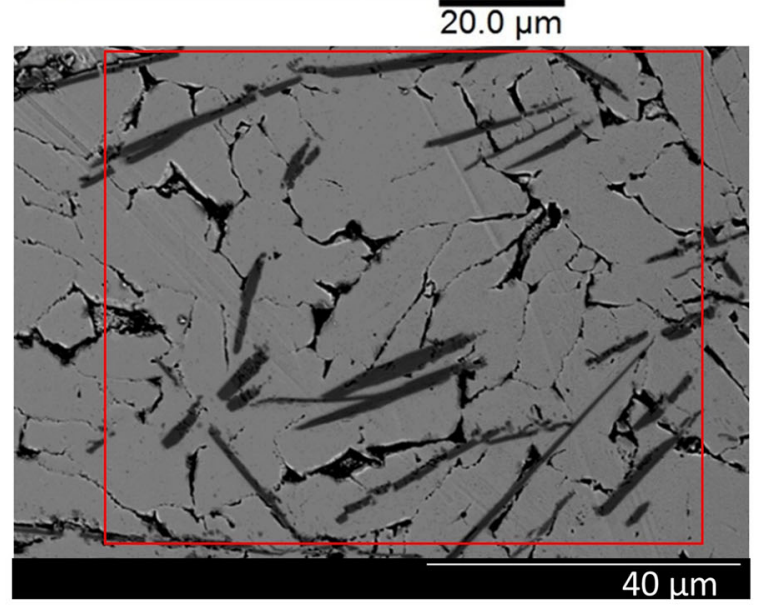

(f)
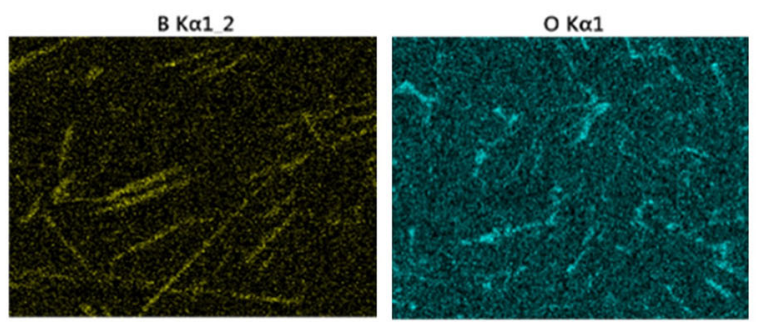

Fig. 6. AFM topography (a, b) and BSE SEM (e, d) images of $0.04 \% \mathrm{~B}(\mathrm{a}, \mathrm{c}, \mathrm{e})$ and $1.09 \% \mathrm{~B}(\mathrm{~b}, \mathrm{~d}, \mathrm{f})$ Ti-6Al-4V samples with corresponding Al and $O(e)$ and $B$ and $O(f)$ EDS maps. Red boxes on the SEM images (c,d) indicate the approximate location of corresponding AFM images (a, b). Pitting visible in the AFM topography images $(a, b)$ shows that corrosion has preferentially occurred within the vanadium-rich metastable $\beta$ phase despite its higher Volta potential. Note that the higher B content sample (b,d,f) exhibits significantly less (and shallower) pitting.

it is kinetically limited, for example, by the presence of a passive layer. Nevertheless, the TiB phase was not found to be an effective cathode in previous studies because the precipitated TiB phase did not decrease or seem to influence corrosion performance under simulated physiological conditions. ${ }^{8}$ Hence, the additions of $\mathrm{B}$ and the presence of the TiB phase appear to be neutral factors regarding corrosion resistance of Ti-6Al-4V + B alloys.

\section{CORROSION BEHAVIOR}

All of the alloys examined here exhibit robust passivity in artificial physiological electrolytes when subjected to the ASTM F2129-15 standard test. ${ }^{7}$
Despite a significant driving force for microgalvanic couple-driven corrosion between the $\beta$ and $\alpha$ phases, any corrosion activity is kinetically limited by the complete passivation of the sample. SKPFM scans conducted on Ti-6Al-4V + B samples after F2129-15 testing showed no significant areas of corrosion attack. Moreover, no significant differences in Volta potential were observed on the surface post-testing as a result of the influence of a uniform passive layer, which effectively homogenized the measured surface potential despite the fact that the phases were still visible in BSE images (Fig. 5). This observation demonstrates that SKPFM is measuring only the surface properties of the surface layer, 
whereas the electron beam interaction volume during SEM interrogates the alloy to a depth beneath the few to tens of nanometer-thick passive film. In addition to the presence of the passivating film on the alloy surface, the surface area fraction of $\beta$ phase relative to $\alpha$ phase may limit the ability of the $\beta$ phase to serve effectively as a cathode under nonforced corrosion conditions.

Under forced corrosion conditions, samples were subjected to polarization scans up to a very high anodic potential ( $8 \mathrm{~V}$ versus $\mathrm{Ag} / \mathrm{AgCl}$ ) in saline solutions. ${ }^{7}$ Localized corrosion was observed to initiate at potentials above $\sim 6.5 \mathrm{~V}$ versus $\mathrm{Ag} / \mathrm{AgCl}$, and the pitting potential decreased slightly with B additions, although the apparent repassivation potential slightly increased. ${ }^{8}$ With the exception of $0.01 \% \mathrm{~B}$, all samples experienced localized corrosion regardless of $\mathrm{B}$ content, although only at potentials well above what would be encountered under physiological conditions. As shown in Fig. 6, corrosion initiation occurred exclusively within the $\beta$ phase regions, as seen in the AFM images (Fig. 6a and b) where the black regions indicate lower height (i.e., areas of dissolution) and are exclusively located where the $\beta$ phase formerly resided surrounding the remaining $\alpha$ grains. Co-located EDS also showed that the remaining surface is passivated by an oxide layer (Fig. 6e and f). Given that the $\beta$ phase is more noble than the $\alpha$ phase and similar in surface potential to the boron-rich precipitates, it is surprising that only the $\beta$ phase was preferentially attacked during the forced corrosion test exposure. The high applied potentials experienced during the test suggest that the $\beta$ phase is both thermodynamically and kinetically unstable above $\sim 6.5 \mathrm{~V}$ versus $\mathrm{Ag} / \mathrm{AgCl}$, resulting in uniform dissolution of the metastable $\beta$ phase, whereas the rest of the microstructure is passivated. Because available thermodynamic data for $\mathrm{Ti}+\mathrm{B}$ and $\mathrm{Ti} 64+\mathrm{B}$ as a function of composition or applied potential are severely limited, computational modeling work is currently underway to construct a Pourbaix diagram and ascertain the relative thermodynamic stability of the phases as a function of $\mathrm{pH}$ and applied potential. Moreover, attack of the $\beta$ phase occurs regardless of wt.\% B composition, and the presence of the precipitated $\mathrm{TiB}$ phase did not appear to have any influence on the preferred location of corrosion propagation, although the higher B content sample showed significantly less pitting and shallower pits, perhaps indicative of a protective effect (see Fig. 6). Ongoing work includes employing an alternative electrolyte (e.g., a reducing solution) to see whether the passivating film can be removed and corrosion initiated under nonforced conditions.

\section{CONCLUSIONS}

The following conclusions can be drawn based on the work reported above:
- The Volta potential difference between $\alpha$ and $\beta$ phases indicates a significant driving force for local galvanic corrosion, but uniform passivation of the alloy surface significantly prevents microgalvanic couple-driven corrosion initiation, including under physiologically relevant conditions.

- Above the B solubility limit, the precipitated TiB phase is noble to both the $\alpha$ and $\beta$ phases, but it does not appear to influence corrosion initiation, propagation, or passivation in electrochemical tests carried out in an artificial physiological electrolyte.

- Under forced corrosion conditions at very high applied potentials, any observed corrosion damage appears limited to preferential dissolution of the $\beta$ phase, regardless of the presence of $B$ or precipitated $\mathrm{TiB}$ phase. The TiB phase did not appear to influence the location for corrosion, and the $\beta$ phase was selectively but uniformly attacked.

- Boron additions do not detrimentally impact overall corrosion resistance or inhibit the robust inherent passivity of Ti-6Al-4V, while offering a significant beneficial effect in regard to the mechanical properties for biomedical implant and device applications. In fact, based on the limited data presented here for forced corrosion, increased boron content may improve corrosion resistance and warrants further study.

\section{ACKNOWLEDGEMENTS}

Assistance from Dr. Nick Bulloss of the Boise State Center for Materials Characterization (BSCMC) with the SEM/EDS work is gratefully acknowledged.

\section{OPEN ACCESS}

This article is distributed under the terms of the Creative Commons Attribution 4.0 International License (http://creativecommons.org/licenses/by/4.0/), which permits unrestricted use, distribution, and reproduction in any medium, provided you give appropriate credit to the original author(s) and the source, provide a link to the Creative Commons license, and indicate if changes were made.

\section{ELECTRONIC SUPPLEMENTARY MATERIAL}

The online version of this article (doi:10.1007/ s11837-017-2378-7) contains supplementary material, which is available to authorized users.

\section{REFERENCES}

1. C.N. Elias, J.H.C. Lima, R. Valiev, and M.A. Meyers, JOM 60,46 (2008).

2. M. Oezcan and C. Haemmerle, Materials 5, 1528 (2012).

3. M.T. Mohammed, Z.A. Khan, and A.N. Siddiquee, Int. J. Chem. Mol. Nucl. Mater. Metal. Eng. 8, 821 (2014). 
4. R.W. Schutz, Corrosion: Materials, eds. S.D. Cramer and B.S. Covino (Materials Park, OH: ASM International, 2005), p. 252.

5. H. Rack and J. Qazi, Mater. Sci. Eng. C 26, 1269 (2006).

6. S. Tamirisakandala, R.B. Bhat, J.S. Tiley, and D.B. Miracle, Scr. Mater. 53, 1421 (2005).

7. V.A. Ravi, S. Rogers, M. Malek, D. Surmenian, I. Priddy, B. Harrison, A. Schissler, S.C. Divi, S. Tamirisakandala, and D. Miracle, JOM 63, 42 (2011).

8. V. Ravi, A. Schissler, W. Chantrjaroen, C. Beecher, A. Razzak, R. Urak, and S. Alas, Corros. Eng. Sci. Technol. 47, 383 (2012).

9. K. McCann, T. Voorhees, G. Margoosian, J. Miguel, and V.A. Ravi, Electrochemical Evaluation of Titanium-Boron Alloys for Potential Biomedical Applications, Paper No. C2016-7842 (Houston, TX: NACE International, 2016).
10. M.F. Hurley, C.M. Efaw, P.H. Davis, J.R. Croteau, E. Graugnard, and N. Birbilis, Corrosion 71, 160 (2015).

11. A. Kvryan, K. Livingston, C.M. Efaw, K. Knori, B.J. Jaques, P.H. Davis, D.P. Butt, and M.F. Hurley, Metals 6, 91 (2016).

12. R.L. Liu, M.F. Hurley, A. Kvryan, G. Williams, J.R. Scully, and N. Birbilis, Sci. Rep. 6, 28747 (2016).

13. C.F. Mallinson and J.F. Watts, J. Electrochem. Soc. 163, C420 (2016).

14. P. Schmutz and G.S. Frankel, J. Electrochem. Soc. 145, 2295 (1998).

15. P. Schmutz and G.S. Frankel, J. Electrochem. Soc. 145, 2285 (1998).

16. D. Nakhaie, A. Davoodi, and G.R. Ebrahimi, Corrosion 72, 110 (2016). 